\title{
Vulnerabilidad a la contaminación del acuífero yucateco bajo escenarios de cambio climático
}

\section{Aquifer pollution vulnerability in Yucatan under climate change scenarios}

\author{
Beth Sua Iztaccihuatl Albornoz-Euán*, Roger Amílcar González-Herrera \\ Facultad de Ingeniería. Universidad Autónoma de Yucatán. Avenida Industrias no contaminantes por Periférico Norte Apartado \\ Postal 150 Cordemex, Mérida, Yucatán, México. \\ ${ }^{*}$ Autor de correspondencia: bethsheva24.1986@gmail.com
}

Artículo científico recibido: 29 de febrero de 2016, aceptado: 09 de noviembre de 2016

RESUMEN. El acuífero yucateco es altamente vulnerable a la contaminación, debido a las características del material geológico, del suelo y las condiciones de recarga; estas pueden modificarse por los cambios del clima. El objetivo de este trabajo fue evaluar la vulnerabilidad a la contaminación del acuífero bajo las variaciones de temperatura y precipitación derivadas del cambio climático. Se usaron los escenarios regionales de cambio climático para México en los horizontes cercano (2015-2039) y futuro (2075-2099), para calcular la evapotranspiración, la recarga y modelar la vulnerabilidad con el índice DRSTIL. Los parámetros del índice DRSTIL son profundidad del agua (D), recarga (R), suelo (S), topografía $(T)$, impacto a la zona vadosa (I) y uso del suelo (L). Cada uno de los parámetros tiene una ponderación de 1 a 5 según su importancia. Los parámetros se dividen en clasificaciones de acuerdo al impacto de contaminación y tienen una puntuación de 1 a 10. La suma ponderada de los factores establecen los valores para los intervalos de clasificación de los grados de vulnerabilidad. Los resultados muestran que bajo los efectos del cambio climático, en comparación con el mapa actual de distribución de áreas vulnerables, se favorece el incremento de la vulnerabilidad moderada, ocupando una mayor extensión y disminución de las superficies de las áreas de alta vulnerabilidad para todos los escenarios de cambio climático, debido al cambio del uso del suelo y la reducción de la recarga.

Palabras clave: Agua subterránea, DRSTIL, escenarios regionales, modelación, recarga

ABSTRACT. The Yucatán aquifer is highly vulnerable to pollution, due to the characteristics of the geological material, soil and recharge conditions, the last of which can be modified by changes in the climate. The aim of this work was to evaluate the aquifer's vulnerability to pollution under the variations of temperature and precipitation caused by climate change. Regional climate change scenarios for Mexico were used in the near (2015-2039) and future (2075-2099) horizons to calculate the evapotranspiration and recharge to model vulnerability with the DRSTIL index. The parameters of the DRSTIL index are water depth (D), recharge (R), soil (S), topography $(T)$, impact to vadose zone (I) and land use (L). Each of the parameters has a weighting of 1 to 5 according to its importance. The parameters are divided into classifications according to the impact of pollution and have a score of 1 to 10 . The weighted sum of the factors establishes the values for the intervals of classification of the degrees of vulnerability. The results show that under the effects of climate change, compared to the current map showing the distribution of vulnerable areas, there is an increase in the area with moderate vulnerability and a decrease in the areas with high vulnerability for all climate change scenarios, due to the change in land use and the reduction in recharge.

Key words: Groundwater, DRSTIL, regional scenarios, modeling, recharge.

\section{INTRODUCCIÓN}

El agua subterránea es un recurso vital para los seres humanos, constituye el principal suministro para más de 1.5 billones de personas alrededor del mundo (Hudack 2005). Pero los acuíferos están experimentando una creciente amenaza de contaminación por la urbanización, el desarrollo in- 


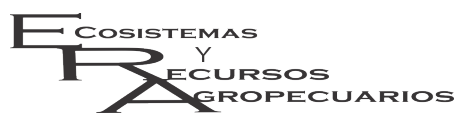

Albornoz-González y González-Herrera Acuífero Yucateco, vulnerabilidad y cambio climático Ecosist. Recur. Agropec. 4(11):275-286,2017

dustrial, las actividades agrícolas y la explotación minera (Foster et al. 2002). Las técnicas para la detección, el monitoreo y la remediación de la contaminación son complejas, costosas y sólo parcialmente efectivas; por lo que se ha dado énfasis a la prevención de la contaminación (Twarakavi y Kaluarachchi 2006).

La modelación y el mapeo de la vulnerabilidad son los primeros pasos en la implementación de programas de gestión del agua subterránea. Hay varios métodos para evaluar la vulnerabilidad a la contaminación del agua subterránea, como el DRASTIC (Aller et al. 1987), GOD (Foster e Hirata 1988), AVI (Van Stempvoort y Evert 1993) y SINTACS (Civita y De Maio 2000). Tradicionalmente estos métodos se implementan en condiciones estáticas, pero la vulnerabilidad depende de factores como la profundidad del nivel freático, la recarga y diversas condiciones de cobertura y uso del suelo, e influyen las variables climáticas y la actividad humana (Li y Merchant 2013).

El cambio climático puede tener impactos relevantes en las aguas subterráneas, por las modificaciones de ciclo hidrológico (IPCC 2007). Por ejemplo, el aumento de la temperatura, la variación en la precipitación y en la evapotranspiración por el calentamiento global pueden ser los principales responsables de las alteraciones en las aguas subterráneas, lo cual puede agravarse por la presión humana, como la extracción insostenible de agua, la irrigación, el cambio de uso del suelo y la urbanización (Pasini et al. 2012). Es necesario considerar, en las estrategias de protección, los efectos de las variaciones de la temperatura y la precipitación, como resultado del cambio climático sobre los acuíferos (Scibek y Allen 2006), para obtener proyecciones fiables del riesgo de contaminación del agua subterránea bajo posibles escenarios futuros (Twarakavi y Kaluarachchi 2006).

Los estudios realizados en el Estado de Yucatán se han enfocado en la vulnerabilidad intrínseca, específicamente en la contaminación por nitratos (Pérez y Pacheco 2004), en lo que se ha aplicado métodos como el DRASTIC, AVI y GOD para la obtención de índices de vulnerabilidad y diferenciación de zonas amenazadas (Pérez et al. 2008). También se reporta la aplicación de DRASTIC en combinación con técnicas estadísticas, análisis multicriterio y SIG para identificar zonas con vulnerabilidad e identificar factores de riesgo (Gijón et al. 2009). En este trabajo se presenta una propuesta de modelación integrando un método de vulnerabilidad basado en el índice DRASTIC (Aller et al. 1987) y los escenarios regionales de cambio climático desarrollados para México. El objetivo fue evaluar la vulnerabilidad a la contaminación del agua subterránea en el Estado de Yucatán bajo las variaciones de temperatura y precipitación derivadas del cambio climático.

\section{MATERIALES Y MÉTODOS}

El área de estudio fue el estado de Yucatán (Figura 1), ocupa el extremo septentrional de la Península del mismo nombre. La geología superficial del estado se compone de caliza dura, formada por la solución y precipitación de carbonato de calcio que cementa granos y fragmentos de concha cerca de la superficie, además de calizas blandas. La falta de arcillas y margas del Terciario Superior sobre la caliza, provoca que en periodos de lluvia se infiltre de forma rápida el agua, disolviendo la roca y formando un relieve llamado karst o cárstico. Debido a las condiciones geológicas el acuífero es considerado como libre, excepto una franja estrecha paralela a lo largo de la costa. El agua subterránea se mueve de las zonas de mayor precipitación ubicadas al sur del Estado, hacia las costas, dispersándose hacia el noroeste, noreste y norte donde se realiza la descarga del acuífero (Graniel 2010).

Para evaluar la vulnerabilidad en el área de estudio se implementó el índice DRSTIL que es una modificación del DRASTIC aplicada por Li y Merchant (2013). Donde D es la profundidad del agua, $\mathrm{R}$ la recarga, $\mathrm{S}$ características del suelo, $\mathrm{T}$ la topografía, I el impacto de la zona vadosa y $L$ el uso del suelo. El subíndice $\mathrm{R}$ es la puntuación y $\mathrm{W}$ el valor de ponderación otorgado a cada factor. El desarrollo de cada uno de los factores del modelo 


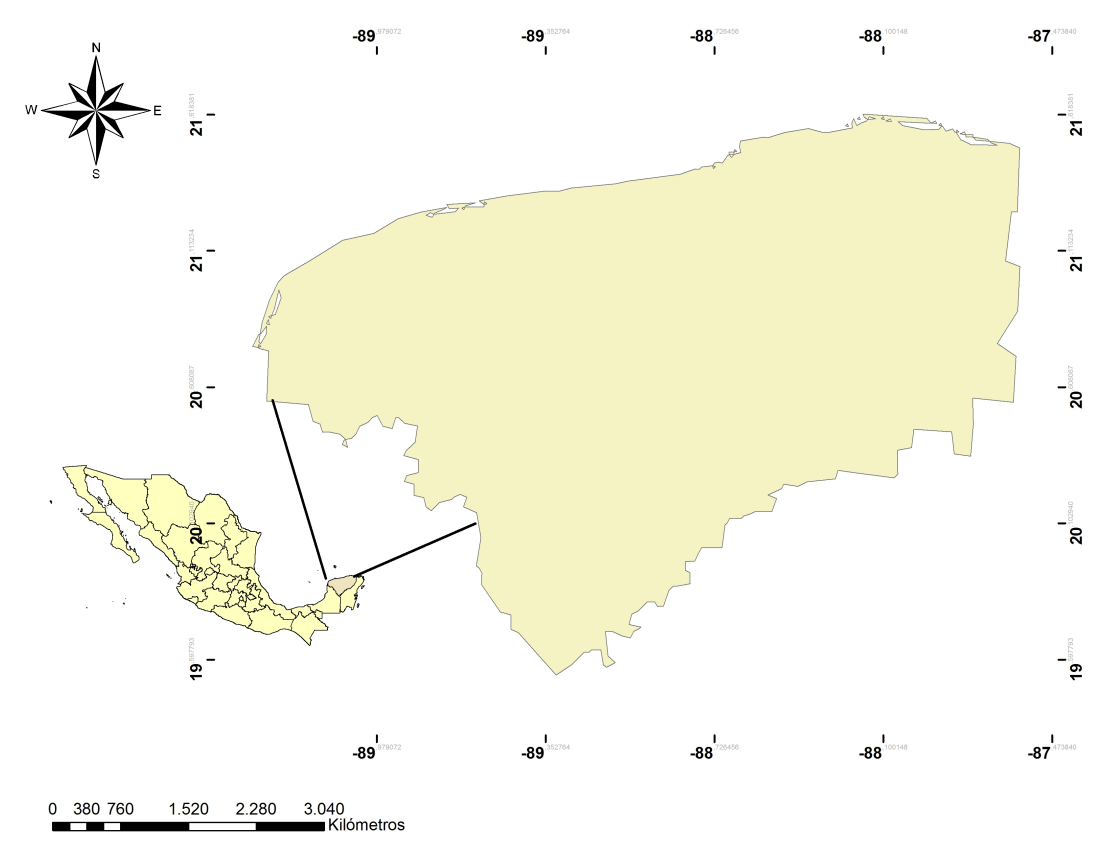

Figura 1. Localización del Estado de Yucatán.

Tabla 1. Fuente de datos para cada factor del índice DRSTIL.

\begin{tabular}{llc}
\hline Factor & \multicolumn{1}{c}{ Fuente de datos } & Ponderación \\
\hline Profundidad del agua & $\begin{array}{l}\text { Bases de datos de la Comisión Nacional del Agua (CONAGUA) } \\
\text { y Modelo Digital de Elevación (MED) del Instituto Nacional de }\end{array}$ & 5 \\
& $\begin{array}{l}\text { Estadística y Geografía (INEGI 2015) } \\
\text { Recarga }\end{array}$ & $\begin{array}{l}\text { Archivos formato shape de la Comisión Nacional para el } \\
\text { Conocimiento y Uso de la Biodiversidad (CONABIO 2015) }\end{array}$ \\
Suelo & $\begin{array}{l}\text { Archivo formato shape de la CONABIO (2015) y guías de inter- } \\
\text { pretación edafológica }\end{array}$ & 2 \\
Topografía & $\begin{array}{l}\text { Modelo Digital de Elevación (MED) del INEGI (2015) } \\
\text { Impacto de la zona } \\
\text { Vadosa Cartas Geológicas y Archivos formato shape de la }\end{array}$ & 5 \\
& $\begin{array}{l}\text { Secretaría de Desarrollo Urbano de Medio Ambiente del Estado } \\
\text { de Yucatán (SEDUMA 2015) }\end{array}$ & \\
Uso de suelo & Archivo formato Shape del INEGI (2015) & 5 \\
\hline
\end{tabular}

DRSTIL se realizó con el programa ArcGIS 10.1.

$$
\underset{L_{R} L_{W}}{D_{R} D_{W}+R_{R} R_{W}+S_{R} S_{W}+T_{R} T_{W}+I_{R} I_{W}+}
$$

En la Tabla 1 se desglosa las fuentes de datos usadas para la obtención de la información para el desarrollo de cada factor del índice DRSTIL, junto con su respectiva ponderación de acuerdo al método DRASTIC (Aller et al. 1987) excepto para el factor uso de suelo, cuyas puntuaciones y ponderación asignada, se tomaron con base en estudios previos de Rupert (1999), Kabbour et al. 2004), Ceplecha et al. 2004), Lima et al. (2011) y Li y Merchant (2013), que incluyen el factor uso del suelo en el índice DRASTIC.

La estimación del factor profundidad del agua se realizó con la metodología de configuración del nivel freático propuesto por Snyder (2008). La cual consiste en combinar las profundidades del nivel freático de las aguas superficiales y subterráneas, así como las elevaciones de un modelo digital de elevación con el objetivo de mejorar la representación del acuífero, debido a la reducción de 


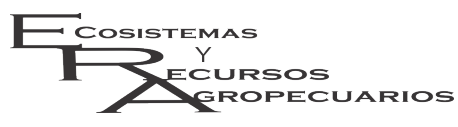

las incertidumbres en las fluctuaciones estacionales del nivel freático. La recarga se calculó con la metodología propuesta por la CONAGUA (2010), donde la recarga natural por infiltración corresponde a la diferencia entre el volumen precipitado menos el volumen evapotranspirado. Los parámetros para el análisis Kriging se determinaron por variogramas desarrollados con el programa GS+.

Para obtener el mapa de vulnerabilidad con el índice DRSTIL, todos los factores se estandarizaron en forma directa a las puntuaciones del método DRASTIC (Aller et al. 1987), con excepción de los factores de Profundidad al Agua (D) y Recarga (R) que se estandarizaron con las ecuaciones derivadas de las curvas de ajuste. Los rásteres estandarizados se agregaron por medio de la Herramienta Suma Ponderada del Módulo Análisis Espacial de ArcGIS 10.1. El ráster de salida se reclasificó de acuerdo con los rangos de valores para el índice DRSTIL calculados por medio de la suma de cada una de las puntuaciones ponderadas para cada factor (Tabla 2).

Tabla 2. Intervalos de clasificación del índice DRSTIL.

\begin{tabular}{ll}
\hline Índice DRSTIL & Clasificación \\
\hline $0-65$ & Mínima \\
$>65-120$ & Baja \\
$>120-160$ & Moderada \\
$>160-192$ & Alta \\
$>192$ & Extrema \\
\hline
\end{tabular}

En la modelación de la vulnerabilidad bajo los efectos de las variaciones de temperatura y precipitación derivados del cambio climático para el Estado de Yucatán, se usaron los escenarios regionales de cambio climático basados en tres Modelos de Circulación General (MCG). Estos modelos simulan una gran variedad de procesos que ocurren en un rango amplio de escalas espaciales y temporales, entre los diversos subsistemas climáticos. Poseen resoluciones espaciales variadas que permiten su aplicación a escalas regionales. Cuando estos modelos se aplican en condiciones de cambio climático dan lugar a la construcción de escenarios de cambio climático para diversas variables,
Albornoz-González y González-Herrera Acuífero Yucateco, vulnerabilidad y cambio climático Ecosist. Recur. Agropec. 4(11):275-286,2017

proyectados a diferentes horizontes (Conde y Gay 2008). Los escenarios son imágenes alternativas de cómo podrían desarrollarse condiciones futuras, es apropiada para analizar cómo las fuerzas motoras influyen en las emisiones futuras y evalúan las incertidumbres asociadas; contribuyendo al análisis del cambio climático (IPCC 2000).

Los MCG utilizados para desarrollar los escenarios regionales fueron el del Instituto Max Planck para la Meteorología (MPI-ESM-LR), el del Laboratorio de Geofísica y Dinámica de Fluidos de la Universidad de Princeton Nueva Jersey (GFDL-CM3) y el de la Red Europea para la Modelación del Sistema Terrestre de la Oficina Meteorológica Centro Hadley (HADGEM2-ES), los cuales se nombran como Modelo Alemán, Modelo Americano y el Modelo Europeo, respectivamente. Cada uno de los MCG se estructuro para tres forzamientos radiativos (RCP): 2.5, 4.5 y 8.5. Para el modelo alemán (HADGEM2-ES) también se consideró el forzamiento radiativo RCP 6.0 .

Los horizontes proyectados para los tres MCG fueron 2015-2039 y 2075-2099 (Cavazos et al. 2013, Fernández et al. 2015). Estos escenarios son de alta resolución espacial, ya que son resultado de un proceso de reducción de escala a $30 " x$ 30 " que equivale aproximadamente a $926 \mathrm{~m} \times 926$ $\mathrm{m}$, e incorporan el efecto topográfico de acuerdo al modelo Shuttle Radar Topography Mission de 90 $\mathrm{m}$ de resolución espacial. Las bases de datos de los escenarios de cambio climático de los MCG en formato GeoTIFF georeferenciados se descargaron de la página del Atlas Climático Digital de México (ACDM) de la Unidad de Informática para las Ciencias Atmosféricas y Ambientales de la Universidad Nacional Autónoma de México (Fernández et al. 2015).

Los archivos en formato GeoTIFF de los tres MCG se recortaron para ajustarlos al área de estudio con ayuda del programa ArcGIS 10.1. Con los datos recortados se extrajeron los promedios mensuales de temperatura media y precipitación para cada RCP de los tres MCG en los dos diferentes horizontes, los cuales sirvieron para calcular la evapotranspiración (ET) y la recarga. La ET y la recarga por infil- 


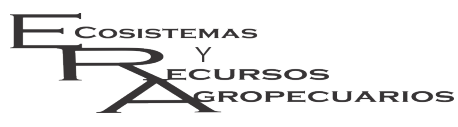

tración se determinaron con un programa codificado en lenguaje Fortran, el cual aplica el Método Turc para el cálculo de la ET y calcula la recarga como la diferencia entre la precipitación anual y la ET.

Los valores de recarga se estandarizaron con respecto a los rangos y las puntuaciones propuestas en el método DRASTIC. Los valores de recarga estandarizados para cada RCP y MCG de los horizontes 2015-2039 y 2075-2099, se interpolaron con el método Kriging en ArcGIS 10.1, de acuerdo a los parámetros obtenidos del variograma del software GS+. Para desarrollar los mapas de vulnerabilidad para cada MCG, los rásteres de recarga interpolados se agregaron a los rásteres de Profundidad (D), Suelo (S), Topografía (T), Impacto de la Zona Vadosa (I) y Uso del Suelo (L) por medio de la herramienta suma ponderada del módulo análisis espacial de ArcGIS 10.1 (ESRI 2010). Los mapas de vulnerabilidad se reclasificaron de acuerdo con los rangos de valores del índice DRSTIL (Tabla 2), los cuales oscilaron entre 17 y 216 , se agrupan para la clasificación en mínima (0-65), baja (> 65-120), moderada $(>120-160)$, alta $(>160-192)$ y extrema (>192).

\section{RESULTADOS}

Los valores del índice de vulnerabilidad DRSTIL en el área de estudio para las condiciones actuales de recarga variaron entre 58 y 197 . En el mapa de vulnerabilidad (Figura 2) se observan las áreas clasificadas con vulnerabilidad mínima que ocupan $1.23 \%$ de la superficie total, se localizan en la región de la Sierrita de Ticul; donde se presentan las mayores profundidades al agua subterránea, pendiente, suelos de textura arcillosa; además de calizas de varios centenares de espesor. La clasificación de vulnerabilidad baja ocupa el $37.95 \%$, se encuentra en la región sur, donde se encuentran acuíferos a mediana profundidad con suelos de texturas margas-arcillosas; en algunas zonas de la región oriente, noreste y el litoral costero donde se localiza estrato confinante con suelos arenosos. En todas estas áreas los valores de recarga no superan los $125 \mathrm{~mm}$. La clasificación de vulnerabilidad con
Albornoz-González y González-Herrera Acuífero Yucateco, vulnerabilidad y cambio climático Ecosist. Recur. Agropec. 4(11):275-286,2017

mayor cobertura fue la moderada con $42.51 \%$ la cual se encuentra en la parte sur de las regiones del oriente, noreste, centro, litoral centro, occidente y poniente de la entidad; en estas zonas se presentan profundidades del agua de 0 a $30 \mathrm{~m}$, los suelos son de textura de margas arcillosas-limosas a arenosas, las calizas son de alta permeabilidad con pendientes del $0 \%$ y la recarga oscila entre los 133 y $280 \mathrm{~mm}$; sin embargo, el uso de suelo es muy variado con zonas de agricultura temporal, pastizal cultivado, pastizal inducido y parches de vegetación natural. Las áreas clasificadas con alta vulnerabilidad ocupan $18 \%$ y se encuentran en la parte central de las regiones del occidente, litoral centro, noreste y en algunas zonas del oriente y el poniente; estas regiones son zonas planas con acuíferos de poca profundidad, los suelos de textura marga limosa a arenosa, con pendientes de $0 \%$, calizas de alta permeabilidad, valores de recarga mayores a $300 \mathrm{~mm}$, con grandes extensiones de agricultura temporal, pastizal cultivado e inducido. La clasificación extrema se presentó en el $0.31 \%$ localizada en la parte sur de la región oriente de la entidad; en esta zona los valores de recarga son superiores a los $300 \mathrm{~mm}$, suelos de textura marga limosa-arenosa, escasa inclinación de las pendientes, calizas de alta permeabilidad y toda el área se encuentra impactada por la agricultura de temporal.

Los valores de recarga de acuerdo a los escenarios regionales de cambio climático para el Estado de Yucatán oscilaran entre 0 y $200 \mathrm{~mm}$ anuales, en contraste con las cantidades actuales de recarga que tienen un rango entre 0 y $491 \mathrm{~mm}$ (Figura 3). Las zonas más afectadas son las tres áreas de mayor recarga que se presentan en el mapa de las condiciones actuales de recarga, que tendrán una disminución hasta del $50 \%$, éstas áreas se encuentran en la parte sur de las regiones noroeste y litoral centro, en la parte norte de la región noreste, en la parte sur y este de la región oriente, y en la porción norte de la región sur del Estado de Yucatán. Los cambios más extremos de recarga se proyectan para los escenarios del Modelo Alemán (MPI-ESM-LR) para el horizonte cercano, con valores que oscilaron entre 0 y $152 \mathrm{~mm}$, para el lejano con valores entre 0 y 138 


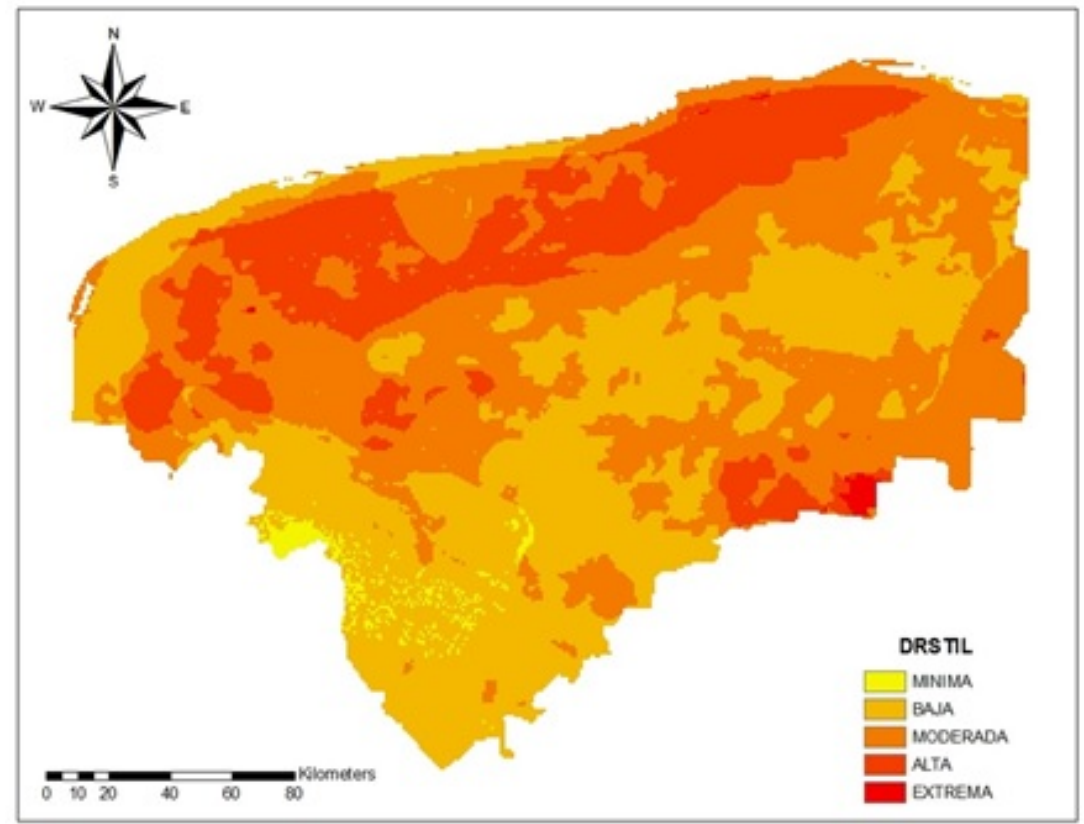

Figura 2. Mapa de vulnerabilidad actual para el Estado de Yucatán.

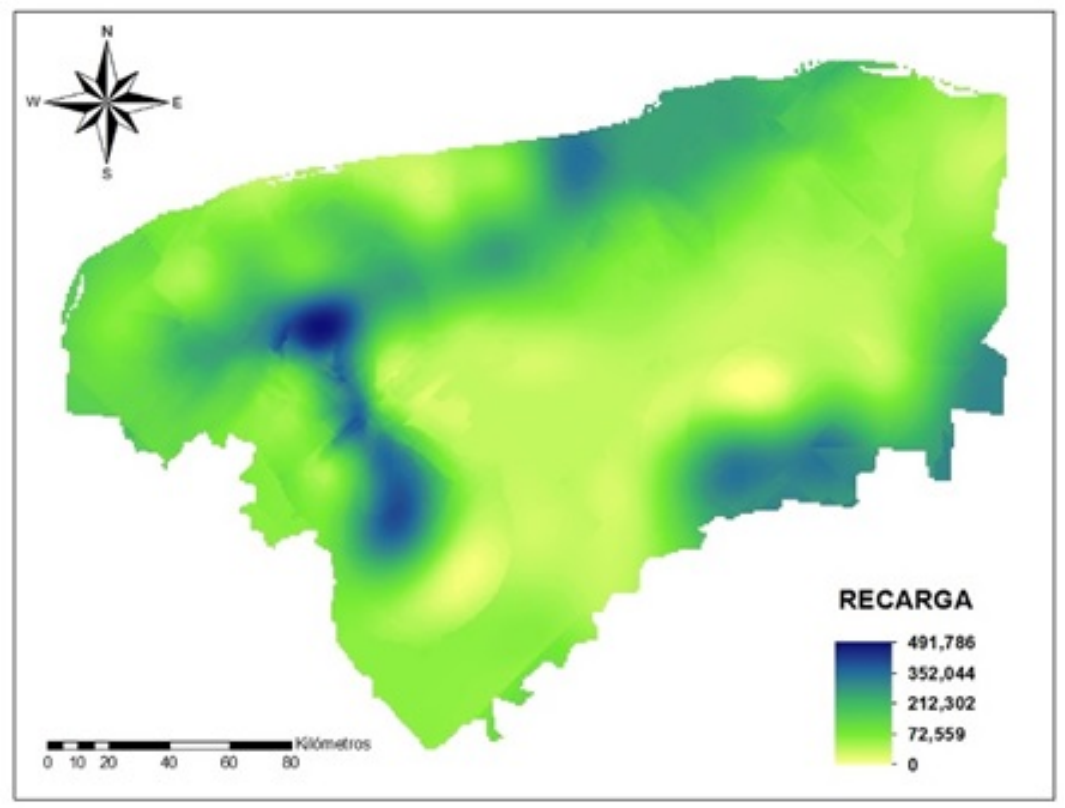

Figura 3. Mapa de recarga actual para el Estado de Yucatán.

$\mathrm{mm}$; siendo este horizonte el forzamiento radiativo RCP 8.5 el que proyectó los valores más bajos con respecto a la recarga futura (Figura 4).

Con base a los valores futuros de la re- carga calculada de los escenarios de los MCG, se desarrollaron los mapas de vulnerabilidad con el índice DRSTIL. Para todos los escenarios, las áreas de alta vulnerabilidad se presentan en las zonas 
del poniente, noroeste, litoral centro, noreste y oriente del estado de Yucatán. Sin embargo, las superficies de dichas áreas variaron entre escenarios de diferentes modelos para distintos horizontes y forzamientos radiativos. En general en todos los escenarios se observa una reducción de las superficies de las áreas de alta vulnerabilidad y un aumento de las áreas de moderada vulnerabilidad, con respecto a las obtenidas en el mapa de vulnerabilidad actual (Tabla 3). Los resultados más extremos se presentan en los mapas del modelo alemán (MPI-ESM$L R$ ), donde las superficies que ocupan las clases de vulnerabilidad alta y moderada, son menores que las de los modelos americano (GFDL-CM3) y europeo (HADGEM2-ES). En el modelo alemán las principales reducciones de las áreas de alta vulnerabilidad se presentan para el forzamiento radiativo RCP 8.5 en el horizonte lejano (Figura 5).

\section{DISCUSIÓN}

Los resultados de la vulnerabilidad a la contaminación del agua subterránea con el índice DRSTIL difieren de los obtenidos en otros trabajos con el índice DRASTIC o variaciones del mismo. Al respecto Pérez y Pacheco (2008) aplicaron el índice DRASTIC para evaluar la vulnerabilidad intrínseca del agua subterránea en el Estado de Yucatán, con resultados que variaron entre 157.6 a 207.8; además dentro de la clasificación se consideraron siete clases: vulnerabilidad insignificante, vulnerabilidad muy baja, baja, moderada, alta, muy alta y extrema. La distribución de las coberturas de cada clase indica que solamente un $0.2 \%$ de la superficie total del estado de Yucatán se encuentra en la categoría de moderada, mientras que el 61 $\%$ se clasificó como categoría alta, el $33.8 \%$ en la categoría muy alta y el $5 \%$ en la categoría extrema. Gijón (2009) aplicó una modificación del DRASTIC (el DSTI) en el cual, a través de análisis de regresión, se determinaron los factores que explican la mayor parte de la variación del índice; de esta manera con el índice DSTI se presentaron solo tres clases de vulnerabilidad: la moderada con $15.48 \%$

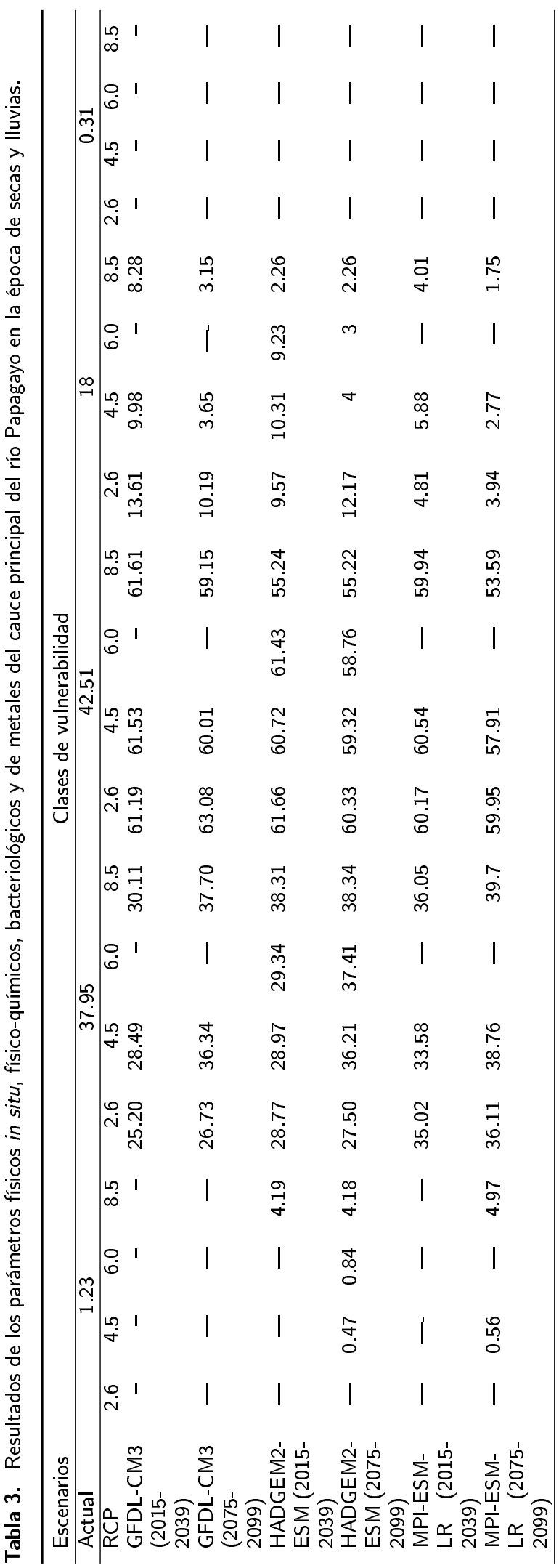




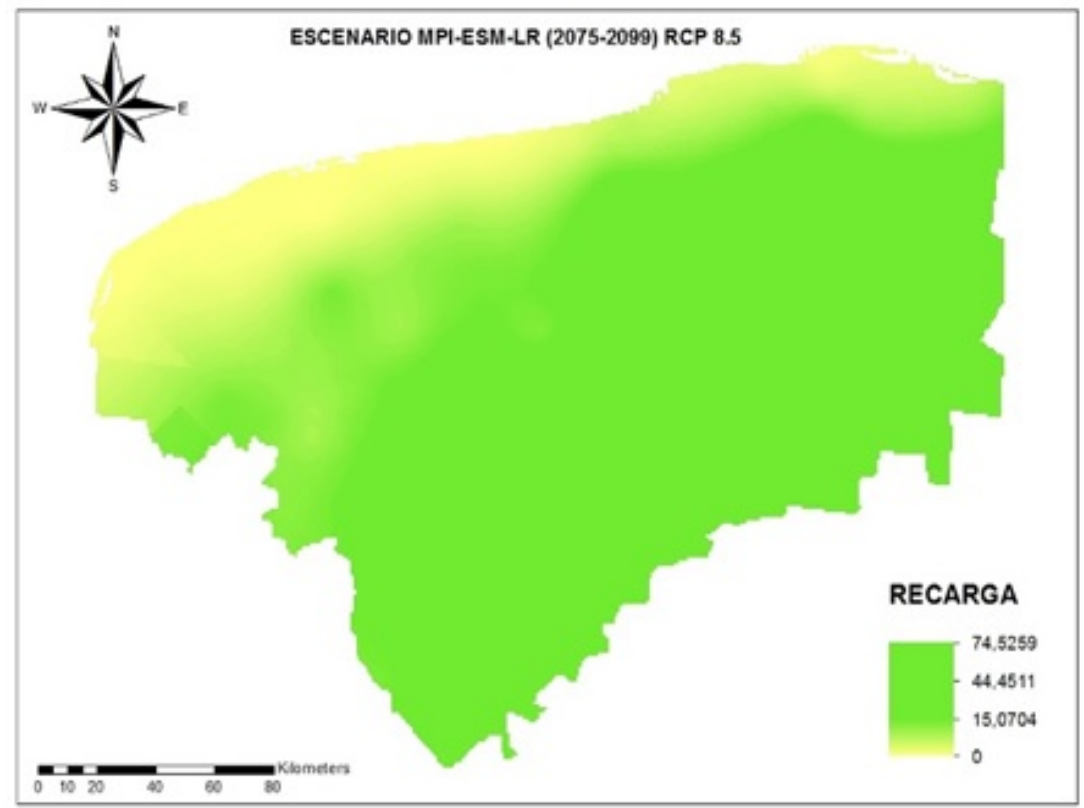

Figura 4. Mapa de recarga futura en el escenario del Modelo Alemán (MPI-ESM-LR) horizonte lejano RCP 8.5.
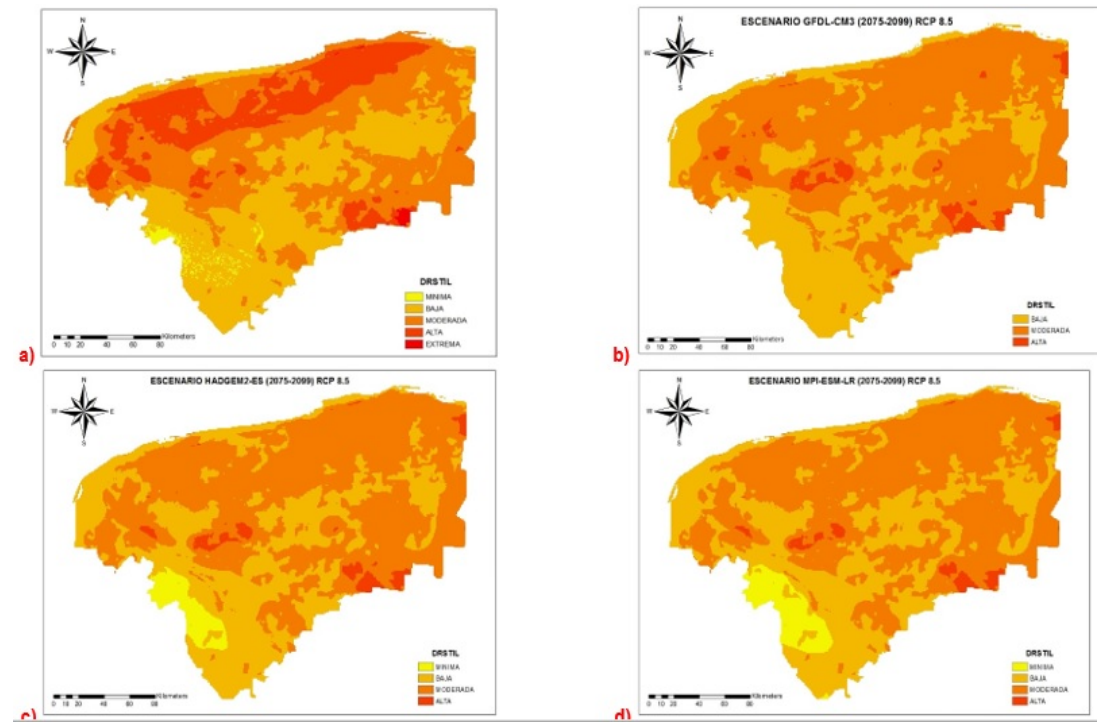

Figura 5. Escenarios de vulnerabilidad futura: a) mapa de condiciones actuales de vulnerabilidad, b) mapa de vulnerabilidad del modelo Americano (GFDL-CM3), c) mapa de vulnerabilidad del modelo Europeo (HADGEM2-ES) y d) mapa de vulnerabilidad según el modelo Alemán (MPI-ESM-LR).

de cobertura respecto al área total, la alta con 35.98 $\%$ y la extrema con $48.54 \%$.

Las variaciones en las distribuciones y coberturas de las clases de vulnerabilidad obtenidas en este trabajo y las reportadas se deben a la aplicación del índice DRSTIL, así como a las diversas fuentes de información y metodologías empleadas para el desarrollo de cada factor del índice. La for- 


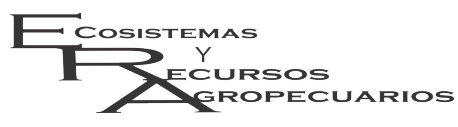

Albornoz-González y González-Herrera Acuífero Yucateco, vulnerabilidad y cambio climático Ecosist. Recur. Agropec. 4(11):275-286,2017

mulación del índice DRSTIL excluye factores como las características del material del acuífero (A) y la conductividad hidráulica (C), ya que estos factores se relacionan con la ruta, longitud, transporte, difusión y degradación de los contaminantes, al considerar todo el sistema del acuífero (Aller et al. 1987). Para el estado de Yucatán, estos dos factores no presentan grandes variaciones en las puntuaciones del esquema de clasificación para cada factor en la metodología de DRASTIC. EI DRSTIL también tiene un factor añadido que es el uso del suelo $(\mathrm{L})$ para reflejar los impactos que las diversas actividades que se desarrollan sobre la superficie del suelo tienen sobre la calidad del agua subterránea; esto es importante debido a que un método que no considera las tendencias del uso del suelo puede subestimar la vulnerabilidad del acuífero para algunas áreas y sobreestimar la vulnerabilidad para otras (Lima et al. 2011). Esta integración permite tener un modelo dinámico con el tiempo, ya que la vulnerabilidad depende de factores como la profundidad del nivel freático, la recarga y las condiciones de la cobertura y uso de suelo (Li y Merchant 2013).

La disminución de las coberturas de las clases alta y extrema, con respecto a las obtenidas en los estudios previos es el resultado de emplear una amplia diferenciación en la textura de los tipos de suelo; en comparación con trabajos anteriores donde el suelo se considera inexistente o delgado para todo el territorio estatal, en esta investigación se consideró la amplia diversidad edáfica de Yucatán que se encuentra representada por la presencia de un conjunto de diversos tipos de suelos (Duch 1991) que se reclasificaron de acuerdo a su textura, una de las propiedades más estables y que determinan el potencial agrícola (White 2006). Al emplear la técnica del balance de las aguas subterráneas de la CONAGUA (2010), se estimó la recarga, como la diferencia entre el volumen precipitado menos el volumen evapotranspirado; lo que permitió obtener una mayor variación en los valores. En la determinación de las profundidades al agua subterránea se usó información de cuerpos de aguas superficiales, de pozos y las elevaciones de un MED para reducir las incertidumbres de las fluctuaciones del nivel freático; se debe de tener en cuenta que el mapa de profundidad es una aproximación y que los valores representan condiciones promedios, debido a que las estimaciones de la profundidad dependen de diversas variables así como de errores asociados a los datos y al método de interpolación (Snyder 2008).

La disminución de la recarga observada en los escenarios de los tres diferentes MCG, se deben a que los efectos esperados del cambio climático en los tres modelos suponen un aumento de la temperatura promedio anual y disminución de la precipitación (Cavazos et al. 2013). Los cambios en la recarga de las aguas subterráneas están determinados por los cambios en la temperatura, evaporación y la precipitación (Kundzewicz et al. 2008). La variación de los rangos de recarga depende del MCG seleccionado; sin embargo, en todos los escenarios, el RCP 8.5 reportó los cambios más extremos. No obstante, esto concuerda con las proyecciones futuras realizadas para el sureste de México bajo el escenario de emisiones RCP 8.5, donde la disminución de la precipitación mensual en la época de lluvias se acentuará, con disminuciones de alrededor de los 30 $\mathrm{mm} \mathrm{mes}^{-1} \mathrm{e}$ incrementos de temperatura extremos de más de cuatro grados (Cavazos et al. 2013).

La modelación de la vulnerabilidad bajo los efectos de la recarga resultado de las variaciones futuras de temperatura y precipitación, favorece el incremento de la vulnerabilidad moderada; esto es debido a la disminución del agua que pueda ser infiltrada, lo que ocasionará cambios en el almacenaje y niveles freáticos del agua subterránea. Por consiguiente, habrá menos probabilidad de arrastre de contaminantes al acuífero. Es necesario indicar que, en la modelación de la vulnerabilidad bajo efectos del cambio climático, sólo se consideró las variaciones futuras de recarga con base en modelos de clima (IPCC 2007). Aunque en la construcción de escenarios regionales se ha logrado reducir de forma significativa las incertidumbres asociadas, es importante considerar forzantes regionales especiales; por ejemplo, el cambio de uso del suelo puede alterar el clima local e incluso regionalmente (McPherson 2007, Magaña 2013). Los escenarios 


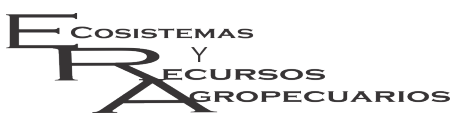

Albornoz-González y González-Herrera Acuífero Yucateco, vulnerabilidad y cambio climático Ecosist. Recur. Agropec. 4(11):275-286,2017

de vulnerabilidad futura desarrollados para el área de estudio basado en los MCG seleccionados presentan un clima más seco pero no incluye la variabilidad climática en periodos cortos de tiempo; hay que tener en cuenta que las variaciones en la temperatura y precipitación asociadas con el ENSO (El Niño-Oscilación del Sur) y la PDO (Oscilación Decadal del Pacífico) influyen en la cantidad de agua que se recarga en los acuíferos y no son representados adecuadamente en los MCG (Toews y Allen 2009, Li y Merchant 2013). Otro aspecto a considerar son los impactos del cambio climático sobre el destino y el transporte de los contaminantes que tienden a ser altamente variables y difícil de predecir debido a las incertidumbres de las proyecciones climáticas (Bloomfield et al. 2006, Li y Merchant 2013). Cualquier estimación de impactos causados por el cambio climático y una adecuada propuesta de adaptación deben sustentarse en el entendimiento de la vulnerabilidad ya que la proyección de esta a dos o tres décadas, con las incertidumbres inherentes a cualquier escenario futuro, constituye la base para estimar cómo el clima puede afectar (Magaña 2013). Los mapas de distribución de la vulnerabilidad representan una aproximación de cómo los cambios futuros de la vulnerabilidad afectan la distribución de la misma.

\section{CONCLUSIONES}

La vulnerabilidad a la contaminación del agua subterránea mostró una diferenciación conservadora, ocupando la vulnerabilidad moderada la mayor extensión. Esto se atribuye al empleo del índice DRSTIL y a las diferentes metodologías aplicadas para el desarrollo de cada uno de los factores. Los resultados de la modelación de la vulnerabilidad a la contaminación del agua subterránea en escenarios de cambio climático, favorecen un aumento en la vulnerabilidad moderada y una reducción de la vulnerabilidad alta. Las variaciones futuras de precipitación y temperatura proyectan una reducción de la recarga del acuífero para la mayor parte del Estado de Yucatán.

\section{AGRADECIMIENTOS}

Al CONACYT por la beca otorgada a la primera autora para realizar estudios de Maestría. Al Cuerpo Académico de Hidráulica e Hidrología y al Cuerpo Académico de Ingeniería Ambiental de la Facultad de Ingeniería de la Universidad Autónoma de Yucatán por el apoyo y la asesoría para la realización del trabajo de investigación. A los revisores por sus valiosas aportaciones y sugerencias para mejorar el artículo.

\section{LITERATURA CITADA}

Aller L, Bennett T, Lehr H, Petty J, Hackett G (1987) DRASTIC: a standardized system for evaluating groundwater pollution potential using hydrogeologic setting. US. Environmental Protection Agency, U.S. Environmental Protection Agency report. EPA/600/2-85/018. Washington, D.C. 622p.

Bloomfield J, Williams R, Gooddy D, Cape J, Guha P (2006) Impacts of climate change on the fate and behaviour of pesticides in surface and groundwater: a UK perspective. Science Total Environmental 369: 163-77.

Cavazos T, Salinas J, Martínez B, Colorado G, De Grau P, Prieto R, et al. (2013) Informe final. Actualización de escenarios de cambio climático para México como parte de los productos de la Quinta Comunicación Nacional. Centro de Ciencias de la Atmósfera UNAM, CICESE, IMTA, INECC, SEMARNAT. 150p.

Ceplecha Z, Waskom R, Bauder T, Sharkoff J, Khosla R (2004) Vulnerability Assessments of Colorado Groundwater to Nitrate Contamination. Water, Air and Soil Pollution 159: 373-394.

Civita M, De Maio M (2000) Valutazione e cartografia automatica de lla vulnerabilità degli acquiferi all'inquinamento con il sistema paramétrico SINTACS R5 a new parametric system for the assessment 
and automatic mapping of groundwater vulnerability to contamination. Pitagora Editrice, Bologna 240p.

CONAGUA (2010) Diagnostico Hídrico de la Región Hidrológica Administrativa XII Península de Yucatán. Gerencia Regional XII Península de Yucatán. SEMARNAT. 592p.

Conde A, Gay C (2008) Guía para la generación de escenarios de cambio climático a escala regional. Centro de Ciencias de la Atmósfera UNAM. México. 104p.

Duch J (1991) La conformación territorial del Estado de Yucatán. Universidad Autónoma de Chapingo. Centro Regional de la Península de Yucatán. 180p.

ESRI (2010) Centro de Recursos de ArcGIS 10.1. http://resources.arcgis.com/es/home/. Fecha de consulta 21 de febrero del 2015.

Fernández E, Zavala H, Romero C, Conde A, Trejo R (2015) Actualización de los escenarios de cambio climático para estudios de impacto, vulnerabilidad y adaptación en México y Centroamérica. UNIATMOS, Centro de Ciencias de la Atmósfera UNAM, INECC. 22p.

Foster S, Hirata R, Gomes D, D’Elia M, Paris M (2002) Protección de la calidad del agua subterránea. Guía para empresas de agua, autoridades municipales y agencias ambientales. Banco Mundial. Washington, DC. $112 p$.

Foster S, Hirata R (1988) Groundwater Pollution Risk Assessment A methodology using available data. Panamerican Center for Sanitary Engineering and Environmental Science (CEPIS). 74p.

Gijón N, Pacheco J, Euán J, Pérez R (2009) Análisis espacial de la vulnerabilidad y riesgo del agua subterránea a la contaminación en el estado de Yucatán México. En: Bautista F, Quintana P, Aguilar Y, Pacheco J, Cabañas D (ed.). Libro del seminario análisis de la vulnerabilidad y riesgo de contaminación de las aguas subterráneas en la península de Yucatán. Centro de Investigaciones en Geografía Ambiental Universidad Nacional Autónoma de México 43p. http://documents.mx/documents/resumenes-vulnerabilidady-riesgos-de-contaminacion-agua subterranea-peninsula-yucatan.html. Fecha de consulta 14 de marzo del 2015.

Graniel E (2010) Hidrología. En: Durán R, Méndez M (ed.). Biodiversidad y desarrollo humano en Yucatán. CICY PPD-FMAM CONABIO SEDUMA. Mérida, Yucatán. pp: 12-13.

Hudack F (2005) Principles of Hydrogeology. Third edition. CRC Press LLC. Florida, USA 248p.

INEGI (2015) Marco Geoestadístico Nacional. http://www3.inegi.org.mx/. Fecha de consulta 20 de enero de 2015.

IPCC (2007) General Guidelines on the use of scenario data for climate impact and adaptation assessment. Task Group on Data and Scenario Support for Impact and Climate Assessment (TGICA). Intergovernmental Panel on Climate Change. 66p. http:// http://www.ipcc-data.org/guidelines/TGICA_guidance sdciaa_v2_final.pdf. Fecha de consulta 27 de enero de 2015.

IPCC (2000) IPCC Special Report Emissions Scenarios Summary for Policymakers. Intergovernmental Panel on Climate Change WHO UNEP 27p. https://www.ipcc.ch/pdf/special-reports/spm/sres-en.pdf. Fecha de consulta 27 de enero de 2015.

Kabbour B, Zouhri L, Mania J, Colbeaux J (2004) Assessing groundwater contamination risk using the DASTI/IDRISI GIS method: coastal system of western Mamora, Morocco. Bulletin of Engineering Geology and the Environment 65: 463-470. 
Kundzewicz ZW, Mata LJ, Arnell NW, Döll P, Jimenez B, Miller K, et al. (2008) The implications of projected climate change for freshwater resources and their management. Hydrological Sciences Journal 53: 3-10.

Li R, Merchant J (2013) Modeling vulnerability of groundwater to pollution under future scenarios of climate change and biofuels-related land use change: A case study in North Dakota, USA. Science of the Total Environment 447: 32-45.

Lima M, Zelaya K, Massone H (2011) Groundwater vulnerability assessment combining the DRASTIC and DYNA-CLUE model in the Argentine Pampas. Environmental Management 47: 828-839.

Magaña V (2013) Guía metodológica para la evaluación de la vulnerabilidad ante cambio climático. Instituto Nacional de Ecología INE Programa de las Naciones Unidas para el Desarrollo PNUD México, DF. 62p.

McPherson RA (2007) A reviews of vegetation-atmosphere interactions and their influences on mesoscale phenomena. Progress in Physical Geography 31: 261-285.

Pasini S, Torresan S, Rizzi J, Zabeo A, Critto A, Marconi A (2012) Climate change impact assessment in Veneto and Friuli plain groundwater. Part II: A spatially resolved regional risk assessment. Science of the Total Environmental 440: 219-235.

Pérez CR, Pacheco AJ (2004) Vulnerabilidad del agua subterránea a la contaminación de nitratos en el estado de Yucatán. Ingeniería 8: 33-42.

Pérez R, Pacheco J, Euán J (2008) Evaluación a Escala Regional de la Vulnerabilidad del Agua Subterránea a la Contaminación en Yucatán, México. Revista Ambiente Ecológico Informes Especiales 14: 1-18.

Rupert M (1999) Improvements to the DRASTIC Groundwater Vulnerability Mapping Method. USGS. http://pubs.usgs.gov/fs/1999/0066/report.pdf. Fecha de consulta 24 de enero de 2015.

Scibek J, Allen DM (2006) Modeled impacts of predicted climate change on recharge and groundwater levels. Water Resources Research 42: 1-18.

Snyder D (2008) Estimated depth to ground water and configuration of the water table in the Portland, Oregon area: US. Geological Survey Scientific Investigations Report 2008-5059, Reston, Virginia. 40p.

Toews MW, Allen DM (2009) Evaluating different GCMs for predicting spatial recharge in an irrigated arid region. Journal of Hydrology 374: 265-281.

Twarakavi NKC, Kaluarachchi JJ (2006) Sustainability of groundwater quality considering land use changes and public health risks. Journal of Environmental Management 81: 405-419.

Van Stempvoort DL, Evert LW (1993) Aquifer vulnerability index: A GIS compatible method for groundwater vulnerability mapping. Canada Water Resources Journal 18: 25-37.

White R (2006) Principles and Practices of Soil Science The soil as a Natural Resource. 4th edition. Blackwell Publishing Company MA, USA. 354p. 